\title{
Dynamic Serosal Perfusion Assessment during Colorectal Resection Using Visible Light Spectroscopy
}

\author{
Alexander Gräfitsch ${ }^{a, b}$ Philipp Kirchhoffa, c Savas D. Soysal ${ }^{a}$ Silvio Däster ${ }^{a}$ \\ Henry Hoffmann ${ }^{\mathrm{a}, \mathrm{c}}$ \\ ${ }^{a}$ General and Visceral Surgery, University Hospital Basel, Basel, Switzerland; bVisceral Surgery, Kantonsspital Aarau, \\ Aarau, Switzerland; ' Center for Hernia Surgery and Proctology, ZweiChirurgen GmbH, Basel, Switzerland
}

\author{
Keywords \\ Colonic perfusion · Spectroscopy · Anastomotic leakage · \\ Colorectal surgery
}

\begin{abstract}
Introduction: Anastomotic leakage (AL) in colorectal surgery occurs with an incidence of up to $20 \%$. Bowel perfusion is deemed to be one of the most important factors for anastomotic healing. However, not much is known about its variability during colorectal surgery and its impact on the outcome. Therefore, this study aims to evaluate serosal oxygen saturation patterns during colorectal resections with visible light spectroscopy (VLS). Materials and Methods: Bowel perfusion in patients undergoing left-sided colorectal resections was assessed at different timepoints during surgery using VLS on the colonic serosa. The primary outcome parameter was serosal oxygen saturation $\left(\mathrm{StO}_{2}\right)$ at the anastomosis during different timepoints of surgery. Results: We included 50 patients who underwent colorectal resection for bowel cancer (58\%) and diverticular disease (34\%). $\mathrm{StO}_{2}$ at the proximal site of the anastomosis increased significantly throughout the surgery (mean difference $3.61 \% ; 95 \% \mathrm{Cl}-6.22$ to $-1.00 ; p=0.008$ ). However, aberrancy from this identified
\end{abstract}

karger@karger.com www.karger.com/esr

Karger $\stackrel{\text { ' }}{5}$

GOPEN ACCESS
(C) 2021 The Author(s)

Published by S. Karger AG, Basel

This is an Open Access article licensed under the Creative Commons Attribution-NonCommercial-4.0 International License (CC BY-NC) (http://www.karger.com/Services/OpenAccessLicense), applicable to the online version of the article only. Usage and distribution for commercial purposes requires written permission. perfusion pattern had no impact on the postoperative outcome. Conclusion: During colorectal resections, we could demonstrate an increase of the colonic $\mathrm{StO}_{2}$ throughout surgery. Appearance of $\mathrm{AL}$ was not associated with lower $\mathrm{StO}_{2}$, underlining the multifactorial genesis of developing AL.

(C) 2021 The Author(s)

Published by S. Karger AG, Basel

\section{Introduction}

Despite the development of multiple techniques to create intestinal anastomoses, anastomotic leakage (AL) still occurs with an incidence of up to $19 \%$ in colorectal surgery [1]. Apart from prolonging hospital stays, it increases the perioperative morbidity, mortality, local tumor recurrence rates, and leads to impaired disease-free survival after oncologic resections [2, 3]. Considering these consequences, we need to understand more about perfusion patterns during colorectal surgery to overcome the lack of accurate prediction of AL in the clinical scenario.

While several factors increase the risk for AL [4], the impact of intestinal perfusion at the level of the colon anastomosis or in remote areas is not fully understood 


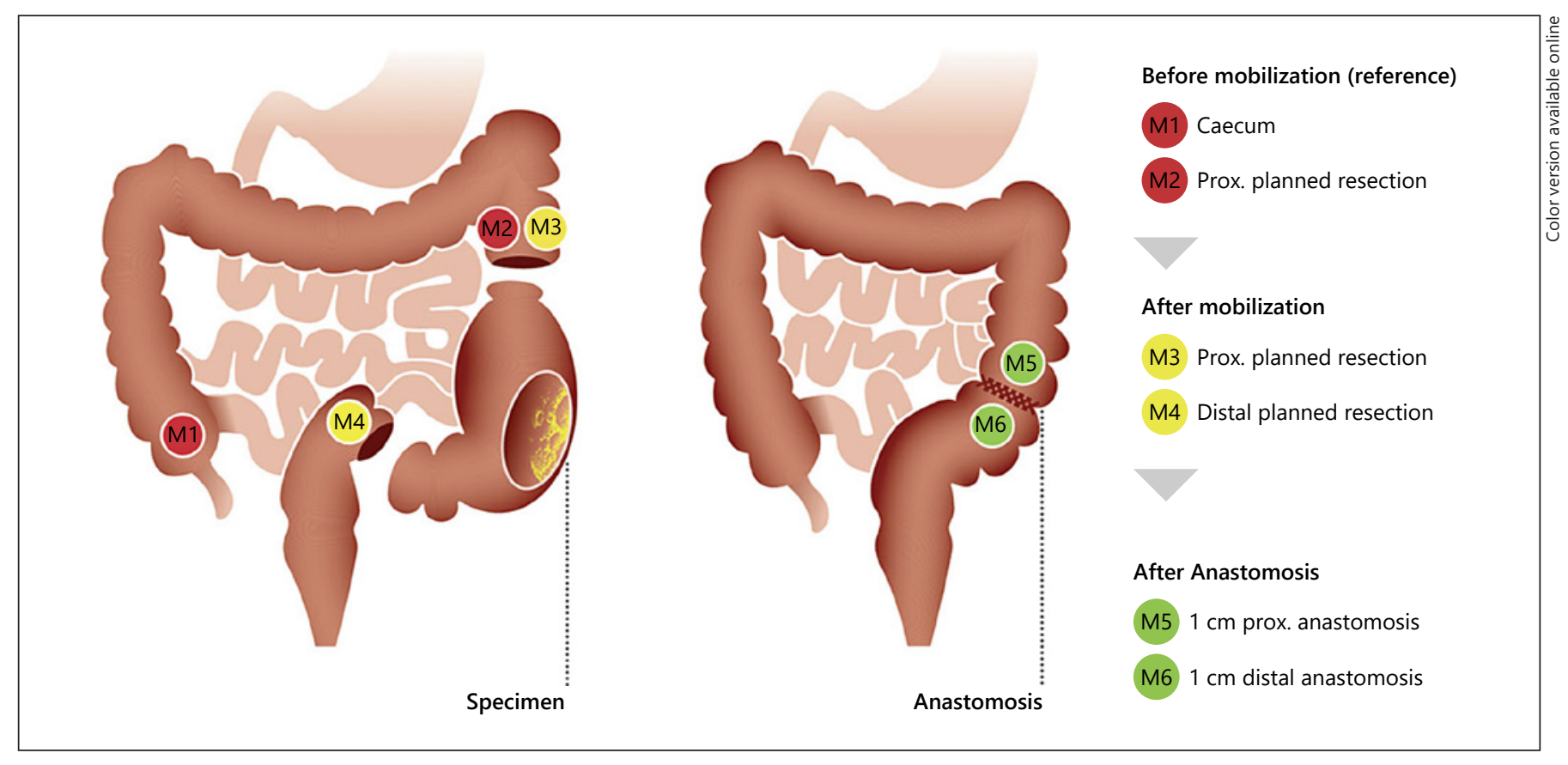

Fig. 1. Measurement protocol. Prox., proximal.

and remains a matter of ongoing debate [5]. Intraoperative assessments of bowel perfusion by palpating mesenteric pulses or evaluating color changes lack any predictive accuracy [6]. Consequently, objective assessments like Doppler sonography, visible light spectroscopy (VLS), indocyanine green (ICG) facilitated narrow-band imaging, and hyperspectral imaging have been introduced [7-9].

Quantification of intraoperative bowel perfusion with VLS is a feasible, non-invasive, and validated technique $[10,11]$. This study aimed to evaluate colonic perfusion patterns during left-sided colonic resections and their correlation to postoperative outcomes.

\section{Materials and Methods}

Patients undergoing left-sided colorectal resections between July 2013 and May 2018 were eligible for participation in this single-center study. Exclusion criteria were colorectal resection at the level of the ascending and transverse colon, age $<18$ years, pregnancy, and patients with special protection regarding the conduction of clinical trials.

Data (demographics, pre- and postoperative variables as well as complications) were collected prospectively and entered anonymously in a database with unique patient identifiers. As anesthetic interventions may influence bowel perfusion during surgery, we also collected parameters like hemoglobin, blood pressure, and catecholamine dosage [12].
We assessed the colonic serosal perfusion during surgery, with a VLS probe (T-Stat ${ }^{\circledR}$, Ischemia Detection System, Spectros Corp., Portola Valley, CA, USA), which is both FDA (US Food and Drug Administration) and CE (Conformité Européenne) certified [13]. The system offers a continuous and non-invasive assessment of the $\mathrm{StO}_{2}$ with normal ranges, provided by the manufacturer, varying from 65 to $77 \%$. The T-Stat ${ }^{\circledR}$ can detect regional and global ischemia as well as reduced-flow and no-flow ischemic states [14].

Following gauze-cleaning of the serosa, we used a 5-mm sensor designed for open and laparoscopic procedures, positioning it a few millimeters above the intestine until the values stabilized within a $5 \%$ margin.

After establishing the pneumoperitoneum in laparoscopic or after adjusting the situs in open surgery, we measured baseline $\mathrm{StO}_{2}$ at the caecum (M1) to evaluate the colon perfusion before any manipulation, as shown in Figure 1. After that, the measurement proximal to the planned oral resection margin was taken (M2). Following this, the mobilization of the bowel, vessel ligation, and resection of the specimen took place. Subsequently, we performed the next assessment at the oral colon margin (M3), the fourth at the aboral colon or rectal stump (M4). Once the anastomosis was formed, we assessed the two final spots, proximal and distal to the anastomosis (M5 and M6).

Since the optimal $\mathrm{StO}_{2}$ for the healing of an anastomosis is unidentified, the anastomosis was only refashioned in cases of gross ischemia. All surgical procedures were performed by board-certified surgeons and senior residents under supervision. We routinely tested colorectal anastomoses with an air-leak test. If positive, the anastomosis was redone with or without defunctioning ileostomy at the surgeon's discretion [15]. After surgery, clinical examinations were conducted on daily ward rounds, and diagnostic tests like computer tomography or endoscopy were carried out as necessary. 
Table 1. Patient characteristics

\begin{tabular}{|c|c|c|c|c|}
\hline & \multicolumn{2}{|l|}{$\mathrm{AL}$} & \multirow[t]{2}{*}{ Total $(n=50)$} & \multirow[t]{2}{*}{$p$ value } \\
\hline & no $(n=42)$ & yes $(n=8)$ & & \\
\hline Age, years & $66.0(57.0-71.0)$ & $66.5(60.0-74.5)$ & $66.0(57.0-73.0)$ & 0.424 \\
\hline Gender male & $29(69.0)$ & $5(62.5)$ & $34(68.0)$ & 0.699 \\
\hline BMI & $23.6(21.3-27.2)$ & $27.2(21.0-32.9)$ & $23.6(21.3-27.5)$ & 0.459 \\
\hline COPD & $5(11.9)$ & $0(0.0)$ & $5(10.0)$ & 0.577 \\
\hline Smoker & $16(38.1)$ & $2(25.0)$ & $18(36.0)$ & 0.694 \\
\hline Pack years & $33.0(17.5-50.0)$ & $17.5(5.0-30.0)$ & $30.0(15.0-50.0)$ & 0.371 \\
\hline ASA & & & & 0.367 \\
\hline 1 & $1(2.4)$ & $0(0.0)$ & $1(2.0)$ & \\
\hline 2 & $22(52.4)$ & $2(25.0)$ & $24(48.0)$ & \\
\hline 3 & $19(45.2)$ & $6(75.0)$ & $25(50.0)$ & \\
\hline Hypercholesterinemia & $8(19.0)$ & $1(12.5)$ & $9(18.0)$ & 1.000 \\
\hline Heart failure & $9(21.4)$ & $2(25.0)$ & $11(22.0)$ & 0.714 \\
\hline Arterial hypertension & $23(54.8)$ & $4(50.0)$ & $27(54.0)$ & 1.000 \\
\hline Diabetes mellitus & $6(14.3)$ & $2(25.0)$ & $8(16.0)$ & 0.598 \\
\hline Hemoglobin, $\mathrm{mg} / \mathrm{mL}$ & $12.9(10.8-14.5)$ & $12.8(10.8-14.3)$ & $12.9(10.8-14.5)$ & 0.863 \\
\hline Hematocrit, \% & $0.4(0.3-0.4)$ & $0.4(0.3-0.4)$ & $0.4(0.3-0.4)$ & 0.618 \\
\hline Laparoscopy & $30(71.4)$ & $6(75.0)$ & $36(72.0)$ & 1.000 \\
\hline Reason for resection & & & & 0.167 \\
\hline Bowel cancer & $25(59.5)$ & $4(50.0)$ & $29(58.0)$ & \\
\hline Diverticular disease & $15(35.7)$ & $2(25.0)$ & $17(34.0)$ & \\
\hline Other & $2(4.8)$ & $2(25.0)$ & $4(8.0)$ & \\
\hline Operation type & & & & 0.842 \\
\hline Left hemicolectomy & $7(16.7)$ & $2(25.0)$ & $9(18.0)$ & \\
\hline Sigmoid colectomy & $29(69.0)$ & $5(62.5)$ & $34(68.0)$ & \\
\hline Low anterior resection & $6(14.3)$ & $1(12.5)$ & $7(14.0)$ & \\
\hline Bowel anastomosis & & & & 0.413 \\
\hline Rectum/colon & $32(76.2)$ & $5(62.5)$ & $37(74.0)$ & \\
\hline Colon/colon & $10(23.8)$ & $3(37.5)$ & $13(26.0)$ & \\
\hline Anastomosis type & & & & 0.313 \\
\hline Hand sewn running & $1(2.4)$ & $1(12.5)$ & $2(4.0)$ & \\
\hline Hand sewn interrupted & $1(2.4)$ & $0(0.0)$ & $1(2.0)$ & \\
\hline Linear stapler device & $2(4.7)$ & $1(12.5)$ & $3(6.0)$ & \\
\hline Circular stapler device & $38(90.5)$ & $6(75.0)$ & $44(88.0)$ & \\
\hline Operation time, min & $200.0(165.0-249.0)$ & $173.5(160.0-248.0)$ & $198.0(160.0-249.0)$ & 0.554 \\
\hline Lowest SBP, mm Hg & $80.0(75.0-90.0)$ & $90.0(87.5-92.5)$ & $85.0(80.0-90.0)$ & 0.018 \\
\hline Highest SBP, mm Hg & $145.0(130.0-160.0)$ & $142.5(135.0-152.5)$ & $145.0(130.0-155.0)$ & 0.633 \\
\hline Lowest $\mathrm{CBT},{ }^{\circ} \mathrm{C}$ & $35.6(35.3-35.9)$ & $35.5(35.3-35.6)$ & $35.6(35.3-35.9)$ & 0.476 \\
\hline Highest CBT, ${ }^{\circ} \mathrm{C}$ & $36.3(36.0-36.6)$ & $36.3(36.0-36.6)$ & $36.3(36.0-36.6)$ & 0.943 \\
\hline Use of inotrope & $30(71.4)$ & $3(37.5)$ & $33(66.0)$ & 0.102 \\
\hline Type of inotrope & & & & 0.211 \\
\hline None & $4(9.5)$ & $2(25.0)$ & $6(12.0)$ & \\
\hline Noradrenaline only & $1(2.4)$ & $0(0.0)$ & $1(2.0)$ & \\
\hline Adrenaline only & $9(21.4)$ & $1(12.5)$ & $10(20.0)$ & \\
\hline Phenylephrine only & $3(7.1)$ & $0(0.0)$ & $3(6.0)$ & \\
\hline Combination & $25(59.5)$ & $5(62.5)$ & $30(60.0)$ & \\
\hline Noradrenaline & $12(28.6)$ & $1(12.5)$ & $13(26.0)$ & 0.662 \\
\hline Noradrenaline dose $\mathrm{e}^{\mathrm{a}}$ & $6.1(3.1-24.0)$ & $87.0(87.0-87.0)$ & $6.3(3.2-33.0)$ & 0.549 \\
\hline Adrenaline & $32(76.2)$ & $5(62.5)$ & $37(74.0)$ & 0.413 \\
\hline Adrenaline, $\mathrm{mg}$ & $22.5(10.0-30.0)$ & $25.0(10.0-35.0)$ & $22.5(10.0-30.0)$ & 0.729 \\
\hline Phenylephrine & $26(61.9)$ & $5(62.5)$ & $31(62.0)$ & 1.000 \\
\hline Phenylephrine, mg & $0.6(0.4-1.1)$ & $1.2(1.0-1.5)$ & $0.6(0.4-1.4)$ & 0.635 \\
\hline
\end{tabular}

Data are presented as the median (IQR) or $n$ (\%). Two-sided $p$ values of Fishers' exact test or Mann-Whitney test, as appropriate. AL, anastomotic leakage; BMI, body mass index; ASA, American Society of Anesthesiologists classification of physical status; SBP, intraoperative systolic blood pressure; CBT, intraoperative core body temperature.

a maximum intraoperative noradrenaline dose $(\mu \mathrm{g} / \mathrm{kg} / \mathrm{min})$.

Dynamic Perfusion Assessment during Colorectal Resection
Eur Surg Res 2021;62:25-31

DOI: $10.1159 / 000514921$ 


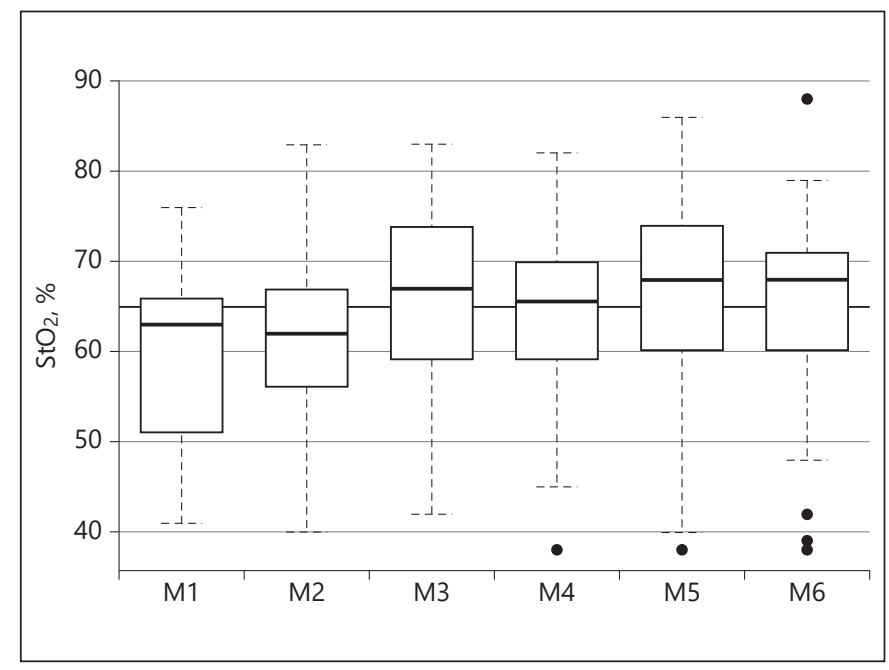

Fig. 2. Whisker plot of serosal saturation during surgery. $\mathrm{StO}_{2}$, serosal capillary oxygen saturation.

Table 2. Development of serosal saturation during surgery

\begin{tabular}{lccl}
\hline & \multicolumn{2}{c}{ AL } & \multirow{2}{*}{$p$ value } \\
\cline { 2 - 3 } & no & yes & \\
\hline M1 & $63(53-67)$ & $53.5(48-63.5)$ & 0.12 \\
M2 & $62.5(55-67)$ & $60.5(57-68.5)$ & 0.92 \\
M3 & $67(59.5-74)$ & $66(49-74)$ & 0.49 \\
M4 & $66(59-71)$ & $65(53-70)$ & 0.83 \\
M5 & $70(60-74)$ & $61(53.5-71.5)$ & 0.27 \\
M6 & $67(60-71)$ & $70(57-72.5)$ & 0.71 \\
\hline
\end{tabular}

Data are the median (IQR). AL, anastomotic leakage.

\section{Statistical Analysis}

Our primary objective was identifying possible perfusion differences before and after anastomosis in left-sided colectomies. Considering results from a previous study, we aimed to include 50 patients with an alpha of 0.05 [11].

We summed up demographics and perioperative categorical data in frequency counts (percentage) and continuous variables with medians (IQR). Differences of perfusion at predefined timepoints (M1-5) are presented as means (95\% CI). We analyzed those differences with paired $t$ tests.

As the primary outcome, we defined $\mathrm{StO}_{2}$ differences proximal to the anastomosis before mobilization and after anastomosis formation (M5 vs. M2). As secondary outcomes, we compared the timepoints M5 vs. M3 and M3 vs. M2. Furthermore, we looked for a possible association between $\mathrm{StO}_{2}$ variations and the occurrence of AL using the Wilcoxon rank-sum test. Statistical analyses were performed using Stata version 14 (StataCorp, College Station, TX, USA) and IBM SPSS Statistics for Windows, version 24 (IBM Corp., Armonk, N.Y., USA).

\section{Results}

\section{Patient and Procedure Characteristics}

We included 50 consecutive patients undergoing leftsided colorectal resections with a median age of 66 years (IQR 60.0-74.5; Table 1). The main indications for surgery were bowel cancer in 29 cases $(58.0 \%)$, followed by diverticular disease in 17 patients (34\%). The most frequent procedures were sigmoid resections $(n=34,68 \%)$ and left hemicolectomies $(n=9,18 \%)$. Overall, the vast majority of procedures were performed laparoscopically $(n=36,72 \%)$. AL occurred in 8 out of 50 patients $(16 \%)$, 2 after open procedures (14.3\%), and 6 after laparoscopic procedures $(16.7 \%)$.

\section{Colon Serosal Oxygen Saturation}

We assessed baseline (M1) $\mathrm{StO}_{2}$ in 49 (49/50, 98\%) patients, with normal $\mathrm{StO}_{2}$ in 18 cases $(18 / 50,37 \%)$. Two patients who developed AL had normal rates at initial measurement at the caecum $(2 / 8,25 \%)$, whereas 25 of 41 (61\%) patients who did not suffer from AL had rates below normal at M1. The median (IQR) $\mathrm{StO}_{2}$ compared at baseline was not significantly different in patients with and without AL (53.5\% [48-63.5] vs. 63\% [53-67], $p=$ 0.12). Similarly, after anastomosis (M5), a non-significant difference was recorded at the oral part of the anastomosis (70\% [60-74] vs. $61 \%$ [53.5-71.5], $p=0.27$; Table 2).

As shown in Figure 2, the overall $\mathrm{StO}_{2}$ rates increased during surgery. We found significant changes comparing timepoints M2 vs. M5 and timepoints M2 vs. M3 (proximal resection level before mobilization vs. after anastomosis and vs. after mobilization) with a mean change of $-3.61 \%(95 \% \mathrm{CI}-6.22$ to $-1.00, p=0.008)$ and $-3.28 \%$ (95\% CI -6.37 to $-0.18 ; p=0.04)$, respectively.

\section{Intra- and Postoperative Parameters}

The median (IQR) hospital stay was 7.0 (5.0-13.0) days, increasing to 25.5 (16.5-32.0) days in the case of $\mathrm{AL}$, which was diagnosed after a median (IQR) of 5 (4.07.5) days. None of the patients with AL had received a loop-ileostomy at the index operation. In 7 cases $(7 / 8$, $87.5 \%)$, surgical management was chosen, with a laparoscopic approach in 3 cases $(3 / 7,42.9 \%)$ and an open approach ( 1 after conversion) in 4 cases $(4 / 7,57.1 \%)$. Endoscopic vacuum therapy was applied in 1 case before and in another after operative revision (2/8, 25\%). One AL was diagnosed after an emergency laparotomy with the formation of an end-ileostomy due to a small bowel lesion. Subsequently, it was managed conservatively after 
a failed attempt to close it endoscopically with an overthe-scope clip $(1 / 8,12.5 \%)$. Twelve patients $(12 / 50,24 \%)$ developed in hospital-related complications with Clavien-Dindo grade III and IV [16]. Patients with AL were more likely to need intensive care unit treatment. Rehospitalization was necessary in 5 patients, resulting in a readmission rate of $9.5 \%(4 / 42)$ in patients without and $12.5 \%(1 / 8)$ in patients with AL.

\section{Discussion}

To the best of our knowledge, this is the first study investigating bowel perfusion focusing on left-sided colorectal resection using VLS at multiple timepoints during surgery to improve the understanding of bowel perfusion patterns and their potential impact on surgical outcomes. While our results show a certain variability of serosal perfusion, we could demonstrate a significant increase throughout surgery. Interestingly, we could not detect significant differences in $\mathrm{StO}_{2}$ between patients with or without subsequent $\mathrm{AL}$, suggesting that serosal perfusion within the normal range does not necessarily protect against developing AL. Likewise, abnormal serosal perfusion at the colon does not necessarily lead to AL. This refutes the frequently proposed assumption that impaired colonic perfusion at the site of the anastomosis increases the risk for developing AL, underlining the multifactorial genesis of this serious postoperative complication following colorectal resection [17].

Although intact bowel perfusion is assumed to be one of the major factors contributing to the healing of colorectal anastomoses, very little is known about the perfusion patterns during the course of the surgery. Even less is known about the bowel perfusion patterns in the postoperative setting since reliable, objective direct measurements are hardly possible [18]. Therefore, we performed multiple measurements at predefined positions and timepoints, to get an impression how bowel perfusion might be impacted during surgery. In contrast, previous studies investigating bowel perfusion concentrated mainly on the anastomotic site alone or performed the measurements only once $[7,10]$. Those approaches ignore the variability of colon perfusion during surgery and may hamper the value of the measurements in the clinical setting.

At baseline (M1), nearly two-thirds of our patients had $\mathrm{StO}_{2}$ levels below normal, with significant improvement at the oral site of the anastomosis throughout sur-

Dynamic Perfusion Assessment during

Colorectal Resection gery (M2 vs. M3 and M2 vs. M5), confirming the results of previous studies [11, 19]. One explanation for this finding could be that bowel manipulation during surgery leads to increased serosal bowel perfusion. However, the impact of anesthetic interventions on intraoperative bowel perfusion is so far hardly understood and a matter of ongoing, controversial discussions. In our patient population, we observed a higher frequency of catecholamine usage in patients who developed an AL. In particular, those patients required higher doses of noradrenaline, adrenaline, and phenylephrine [20].

Typical risk factors, such as higher body mass index or a higher American Society of Anesthesiologists (ASA) score, were more common in patients who developed an AL, reflecting the results of other studies [1]. Regarding the possible influence of the surgical approach on AL rates, we did not see a difference in leakage rates between open and laparoscopic procedures.

Our findings underline the multifactorial pathogenesis of AL. Low intraoperative blood pressure, liberal perioperative fluid management, and the gut microbiome seem to contribute to its occurrence $[21,22]$. Consequently, a trial protocol assessing perfusion as well as multiple other factors, such as mechanic stability and tension, as risk factors for AL was recently published [23].

While some trials have described lower AL rates due to utilization of ICG [24], other publications did not see different rates [25]. Hyperspectral imaging results led to changes of resection planes in one study; however, further studies are needed to determine a possible impact on AL [9]. In our cohort, perfusion did not differ significantly at any point during surgery between patients with or without subsequent AL. Thus, we conclude that intraoperative measurement of the bowel perfusion alone does not reduce AL rates and therefore may not add any additional value.

There are some limitations to the study, which we would like to address. The chosen sample size is relatively small but reflects sample sizes of comparable studies focusing on this topic. Moreover, despite concentrating on left colonic and colorectal resections, there is still a wide variety of included procedures. Consequently, multivariate analyses to assess other known risk factors were not feasible in our study. Lastly, one may argue that the pinpoint approach of measurement with the T-Stat ${ }^{\circledR}$ probe does not truly reflect the perfusion of the whole region and that environmental effects like light pollution or stained body fluids could have interfered with the measurements. However, as previous publications have 
shown, this approach delivers reliable results when measuring the colonic perfusion [11].

In summary, our study contributes to the understanding of bowel perfusion patterns during colorectal surgery and their impact on the clinical outcome. Apart from a significant increase of $\mathrm{StO}_{2}$ of the colon over the course of the surgery, we were unable to demonstrate an association between impaired colon perfusion and the risk for developing AL. This underlines the multifactorial genesis of AL and shifts the focus away from solitary evaluation of the bowel perfusion towards an interdisciplinary management.

\section{Statement of Ethics}

The present study was conducted according to the regulations of the Swiss federal act on research involving human beings (Human Research Act, HRA) and the guidelines of good clinical practice (GCP). The local Ethical Committee (Ethikkommission beider Basel, EKBB) approved the trial before its start (ref. No. EK 316/12) and written informed consent was obtained from all participants.

\section{Conflict of Interest Statement}

The authors have no conflicts of interest to declare.

\section{Funding Sources}

This study was funded by the Voluntary Academic Society (Freiwillige akademische Gesellschaft - FAG) Basel (Switzerland) and the Gottfried and Julia Bangerter-Rhyner Foundation, Basel (Switzerland). The Basel Institute for Clinical Epidemiology and Biostatistics is supported by grants from Santé Suisse and the Gottfried and Julia Bangerter-Rhyner Foundation.

\section{Author Contributions}

A.G. was in charge of the database and contributed by writing the manuscript. S.D. and S.D.S. acted as proofreaders and collected data. P.K. and H.H. were responsible for the study conception and design, served as proofreaders, and collected data.

\section{References}

1 Sciuto A, Merola G, De Palma GD, Sodo M, Pirozzi F, Bracale UM, et al. Predictive factors for anastomotic leakage after laparoscopic colorectal surgery. World J Gastroenterol. 2018 Jun;24(21):2247-60.

2 Choi HK, Law WL, Ho JW. Leakage after resection and intraperitoneal anastomosis for colorectal malignancy: analysis of risk factors. Dis Colon Rectum. 2006 Nov;49(11): 1719-25.

3 Gessler B, Eriksson O, Angenete E. Diagnosis, treatment, and consequences of anastomotic leakage in colorectal surgery. Int $\mathrm{J}$ Colorectal Dis. 2017 Apr;32(4):549-56.

4 Frasson M, Flor-Lorente B, Rodríguez JL, Granero-Castro P, Hervás D, Alvarez Rico MA, et al.; ANACO Study Group. Risk factors for anastomotic leak after colon resection for cancer: multivariate analysis and nomogram from a multicentric, prospective, national study with 3193 patients. Ann Surg. 2015 Aug;262(2):321-30.

5 Shogan BD, Carlisle EM, Alverdy JC, Umanskiy K. Do we really know why colorectal anastomoses leak? J Gastrointest Surg. 2013 Sep;17(9):1698-707.

6 Karliczek A, Harlaar NJ, Zeebregts CJ, Wiggers T, Baas PC, van Dam GM. Surgeons lack predictive accuracy for anastomotic leakage in gastrointestinal surgery. Int J Colorectal Dis. 2009 May;24(5):569-76.
7 Wada T, Kawada K, Takahashi R, Yoshitomi M, Hida K, Hasegawa S, et al. ICG fluorescence imaging for quantitative evaluation of colonic perfusion in laparoscopic colorectal surgery. Surg Endosc. 2017 Oct;31(10):418493.

8 Kream J, Ludwig KA, Ridolfi TJ, Peterson CY. Achieving low anastomotic leak rates utilizing clinical perfusion assessment. Surgery. 2016 Oct;160(4):960-7.

9 Jansen-Winkeln B, Germann I, Köhler H, Mehdorn M, Maktabi M, Sucher R, et al. Comparison of hyperspectral imaging and fluorescence angiography for the determination of the transection margin in colorectal resections-a comparative study. Int J Colorectal Dis. 2021 Feb;36(2):283-91.

10 Karliczek A, Benaron DA, Baas PC, Zeebregts CJ, Wiggers T, van Dam GM. Intraoperative assessment of microperfusion with visible light spectroscopy for prediction of anastomotic leakage in colorectal anastomoses. Colorectal Dis. 2010 Oct;12(10):101825.

11 Hoffmann H, Delko T, Kirchhoff P, Rosenthal R, Schäfer J, Kraljević M, et al. Colon perfusion patterns during colorectal resection using visible light spectroscopy. World J Surg. 2017 Nov;41(11):2923-32.

12 Nygren A, Thorén A, Ricksten SE. Vasopressors and intestinal mucosal perfusion after cardiac surgery: norepinephrine vs. phenylephrine. Crit Care Med. 2006 Mar;34(3):7229.
13 Spectros Medical Devices Inc. [Internet] Norfolk: Product information T-Stat ${ }^{\circledR}$ [cited December 15, 2019]. Available from: spectros.com/index.php?id=55.

14 Delko T, Hoffmann H, Kraljević M, Droeser RA, Rothwell L, Oertli D, et al. Intraoperative patterns of gastric microperfusion during laparoscopic sleeve gastrectomy. Obes Surg. 2017 Apr;27(4):926-32.

15 Kryzauskas M, Degutyte A, Abeciunas V, Lukenaite B, Jasiunas E, Poskus E, et al. Experimental study of mechanical integrity testing in stapled large bowel: methylene blue leak test is not inferior to air leak test. Visc Med. 2020 Oct;1-9.

16 Dindo D, Demartines N, Clavien PA. Classification of surgical complications: a new proposal with evaluation in a cohort of 6336 patients and results of a survey. Ann Surg. 2004 Aug;240(2):205-13.

17 Foppa C, Ng SC, Montorsi M, Spinelli A. Anastomotic leak in colorectal cancer patients: new insights and perspectives. Eur J Surg Oncol. 2020 Jun;46(6):943-54.

18 Sherwinter DA. Transanal near-infrared imaging of colorectal anastomotic perfusion. Surg Laparosc Endosc Percutan Tech. 2012 Oct;22(5):433-6. 
19 Karliczek A, Benaron DA, Baas PC, Zeebregts CJ, van der Stoel A, Wiggers T, et al. Intraoperative assessment of microperfusion with visible light spectroscopy in esophageal and colorectal anastomoses. Eur Surg Res. 2008;41(3):303-11.

20 Hiltebrand LB, Koepfli E, Kimberger O, Sigurdsson GH, Brandt S. Hypotension during fluid-restricted abdominal surgery: effects of norepinephrine treatment on regional and microcirculatory blood flow in the intestinal tract. Anesthesiology. 2011 Mar;114(3):55764.
21 van Rooijen SJ, Huisman D, Stuijvenberg M, Stens J, Roumen RMH, Daams F, et al. Intraoperative modifiable risk factors of colorectal anastomotic leakage: Why surgeons and anesthesiologists should act together. Int J Surg. 2016;36(Pt A):183-200.

22 Lauka L, Reitano E, Carra MC, Gaiani F, Gavriilidis P, Brunetti F, et al. Role of the intestinal microbiome in colorectal cancer surgery outcomes. World J Surg Oncol. 2019 Dec;17(1):204.

23 Kryzauskas M, Poskus E, Dulskas A, Bausys A, Jakubauskas M, Imbrasaite U, et al. The problem of colorectal anastomosis safety. Medicine. 2020 Jan;99(2):e18560.
24 Alekseev M, Rybakov E, Shelygin Y, Chernyshov S, Zarodnyuk I. A study investigating the perfusion of colorectal anastomoses using fluorescence angiography: results of the FLAG randomized trial. Colorectal Dis. 2020 Sep;22(9):1147-53.

25 De Nardi P, Elmore U, Maggi G, Maggiore R, Boni L, Cassinotti E, et al. Intraoperative angiography with indocyanine green to assess anastomosis perfusion in patients undergoing laparoscopic colorectal resection: results of a multicenter randomized controlled trial. Surg Endosc. 2020 Jan;34(1):53-60. 